\title{
Re-Examining TID Hardness Assurance Test Protocols for SiGe HBTs
}

\author{
Peng Cheng, Student Member, IEEE, Jonathan A. Pellish, Member, IEEE, Martin A. Carts, Member, IEEE, \\ Stanley Phillips, Student Member, IEEE, Edward Wilcox, Student Member, IEEE, \\ Tushar Thrivikraman, Student Member, IEEE, Laleh Najafizadeh, Student Member, IEEE, \\ John D. Cressler, Fellow, IEEE, and Paul W. Marshall, Member, IEEE
}

\begin{abstract}
We investigate the applicability of current total ionizing dose (TID) test protocols in the context of advanced transistor technologies such as Silicon-Germanium heterojunction bipolar transistors (SiGe HBTs). In SiGe HBTs, an unexpected shift in collector current is observed during total dose irradiation. Using both device and circuit measurements, we investigate this phenomenon and assess its potential importance in hardness assurance of $\mathrm{SiGe}$ components. TCAD simulations were performed to explain the observed current shifts.
\end{abstract}

Index Terms-Anneal, BGR, dose rate, gamma, Gummel, phaseshifter, proton, Silicon-Germanium heterojunction bipolar transistors (SiGe HBTs).

\section{INTRODUCTION}

$\mathbf{T}$ $\mathrm{O}$ ensure adequate on-orbit reliability, space electronics are extensively pre-flight tested under various radiation environments. One such reliability concern is total ionizing dose (TID), in which charge is accumulated in devices over time, inevitably degrading the performance of the transistors and the circuits built from them. Standard TID testing protocols for hardness assurance of space-borne military and aerospace electronics systems exist [1], and generally follow a testing sequence consisting of: 1) measure the target device-under-test (DUT) before radiation exposure, 2) irradiate the DUT to a certain total dose, and 3) re-measure the DUT in a radiation-free environment to quantify the impact of the radiation exposure. In practice, however, there is a time lapse between steps 2 and 3 , and as a result critical information could be potentially lost

Manuscript received July 17, 2009; revised September 08, 2009. Current version published December 09, 2009. This work was supported in part by the Defense Threat Reduction Agency under the Radiation Hardened Microelectronics Program, an AFOSR MURI program, NASA-GSFC under the NASA Electronic Parts and Packaging (NEPP) Program, NASA ETDP, and in part by IBM

P. Cheng, S. Phillips, E. Wilcox, T. Thrivikraman, L. Najafizadeh, J. Yuan, and J. D. Cressler are with the School of Electrical and Computer Engineering, Georgia Institute of Technology, Atlanta, GA 30332-0250 USA (e-mail: pcheng@ece.gatech.edu).

J. A. Pellish is with the NASA/GSFC Radiation Effects and Analysis Group, Greenbelt, MD 20771 USA.

M. A. Carts is with MEI Technologies, NASA/GSFC Radiation Effects and Analysis Group, Greenbelt, MD 20771 USA.

P.W. Marshall is with NASA-GSFC, Brookneal, VA 24528 USA.

Color versions of one or more of the figures in this paper are available online at http://ieeexplore.ieee.org.

Digital Object Identifier 10.1109/TNS.2009.2032857 due to the complex time-dependent nature of charge dynamics in silicon devices [2]. This is why Section 3.10 of the military standard MIL-STD-883G Test Method 1019.7 [1] particularly recommends that the time from end of irradiation to start of electrical measurement be as short as possible, and that maximum delay time should be 1 hour (for regular radiation testing). If any radiation-induced damage mechanisms are missed in such TID qualification testing, the impact to mission lifetime can be potentially significant. Given the complexity of mixed-signal circuits and space radiation physics, it would seem prudent to re-evaluate this common practice, particularly as it relates to new, more advanced devices technologies such as Silicon-Germanium heterojunction bipolar transistors ( $\mathrm{SiGe}$ HBTs) that have emerged in recent years.

SiGe HBTs have been extensively tested for radiation hardness assurance for space applications, as can be seen in the numerous publications in the past decade. Performed under the standard test procedure, virtually no degradation in collector current $\left(I_{C}\right)$ post-exposure [3], [4] is observed, whereas the base current $\left(I_{B}\right)$ typically shows a near $2 k T$ slope leakage component in the base current. A typical pre-irradiation and post-irradiation Gummel characteristic is shown in Fig. 1 to illustrate. Many studies have been focused on the degradation of base current, since it affects common device figures-of-merit such as current and RF gain [4].

The question still remains, though, as to whether the current testing protocol underestimates degradation due to the annealing that potentially occurs in the time lapse between steps 2 and 3. In this paper, we explore this question by conducting TID testing in a manner that differs from the traditional total dose testing protocol: device and circuit terminal currents were monitored in real-time before, during, and after irradiation, so that no time gap exists between exposure and re-measurement. Using our new testing method, we observed excess $I_{C}$ in addition to $I_{B}$ leakage during exposure. In order to better gauge the severity and the underlying mechanism behind the excess $I_{C}$, proton and gamma irradiation at a wide range of dose rates were performed. Several SiGe technology platforms were tested, at different bias conditions, to confirm whether this is a process-specific issue.

Excess $I_{C}$ has been previously observed in BJTs [5], [6]; however, for aggressively scaled SiGe HBTs the picture remains unclear. Indeed, $I_{C}$ degradation has been observed in SiGe HBTs, but its response to total dose and dose rate are still not well-understood [7]-[9]. Using our new measurement approach, radiation-induced changes in $I_{C}$ were monitored 


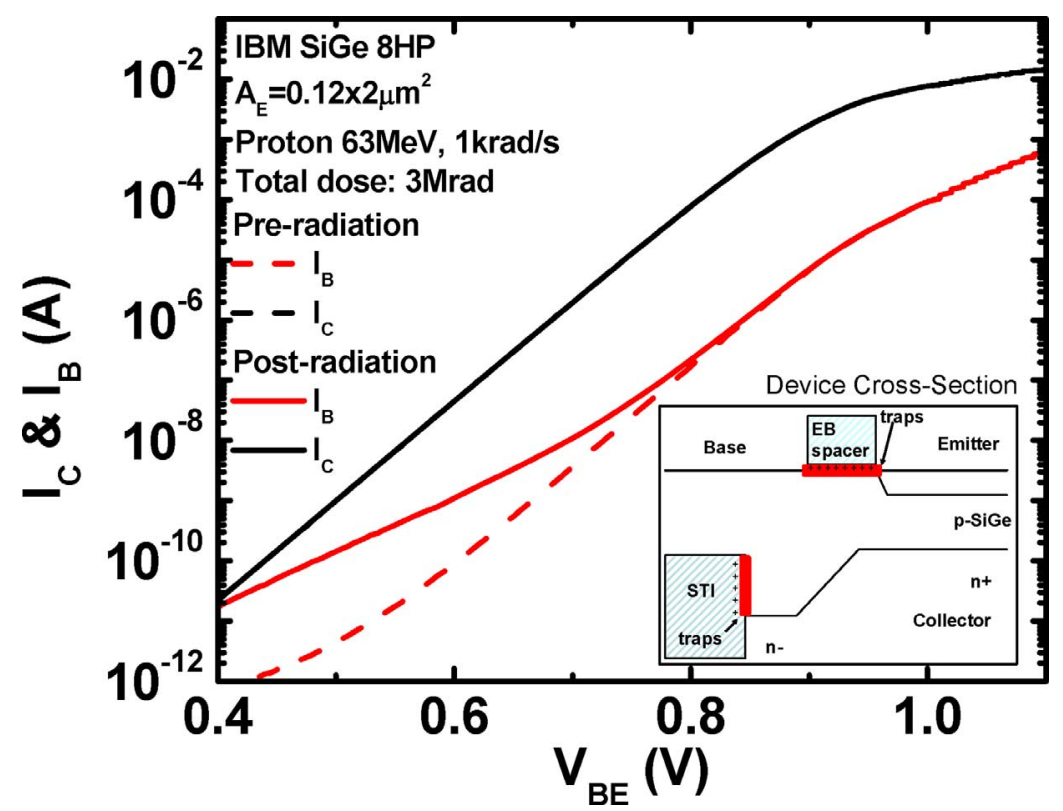

Fig. 1. Typical pre- and post-irradiation Gummel characteristics, showing that $I_{C}$ remains unchanged but $I_{B}$ is increased due to excess $2 k T$ recombination component.

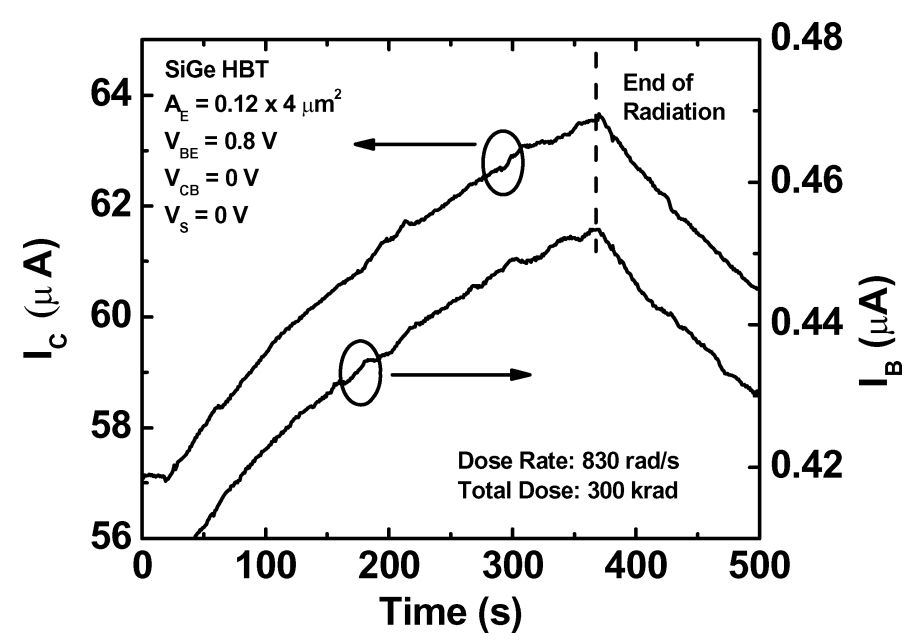

Fig. 2. Collector and base currents of a SiGe HBT under irradiation of 300 krad. Radiation stopped at $360 \mathrm{~s}$, but biasing continued for $140 \mathrm{~s}$.

continuously, allowing very repeatable trends to be demonstrated and understood.

SiGe BiCMOS circuits were also irradiated in order to probe the potential coupling between transistor-level effects and circuit and system effects. In this case, the bias currents of selected $\mathrm{SiGe}$ analog and RF circuits were also monitored before, during, and after irradiation to assess the impact at the circuit level. An explanation for this excess $I_{C}$ phenomenon and its implication to orbital electronic circuit design are discussed.

Hardness assurance results from this paper reveal differences when compared with the literature, which we believe raise fundamental questions about the applicability of current hardness assurance procedures for SiGe HBT technologies, and these implications will also be discussed.

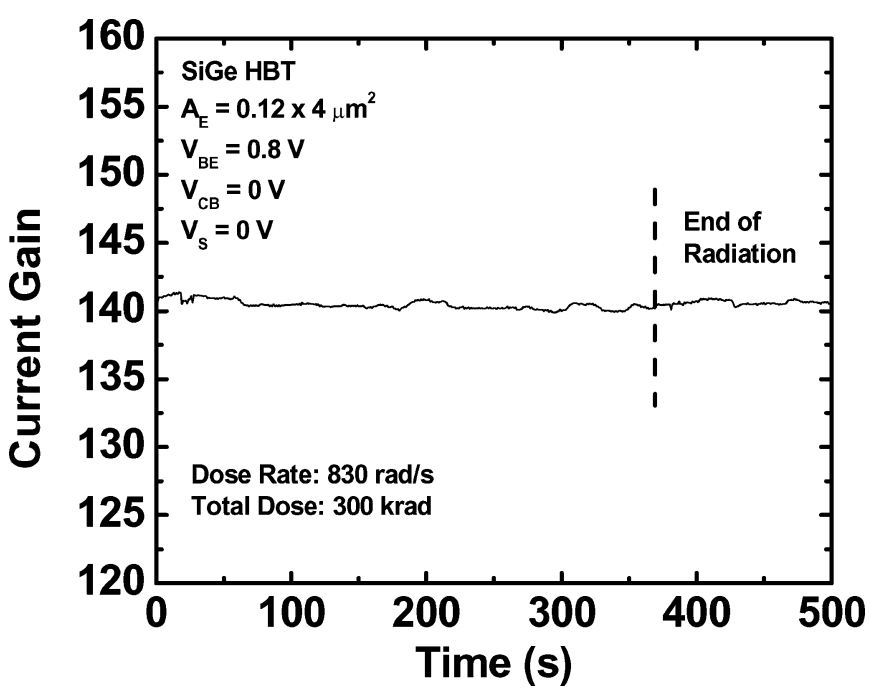

Fig. 3. Current gain for the SiGe HBT from Fig. 4, for a total dose irradiation of $300 \mathrm{krad}(\mathrm{Si})$.

\section{DEVICES AND TeSt CONDITIONS}

The devices and circuits used in this study are from several SiGe BiCMOS technologies. One of them was IBM's SiGe $8 \mathrm{WL}$ technology, which is a cost-performance variant of the $130 \mathrm{~nm}$ node high-performance platform technology [10]. IBM $5 A M$ [11], with peak cut-off frequency of $50 \mathrm{GHz}, 0.5 \mu \mathrm{m}$ SiGe process, was used for gamma irradiation. Samples were diced and packaged into 28-pin dual-inline packages, with two or three devices bonded out in each package. SiGe HBT and CMOS phase shifters were fabricated in the IBM SiGe 8HP process [12]. While both types of X-band $(8 \mathrm{GHz}-12 \mathrm{GHz})$ phase shifters (PS) use a switched high/low-pass filter topology, one circuit uses SiGe HBTs in diode-connected configurations for the RF switch, while the other circuit uses CMOS devices in pass-gate configuration. Precision bandgap voltage references 


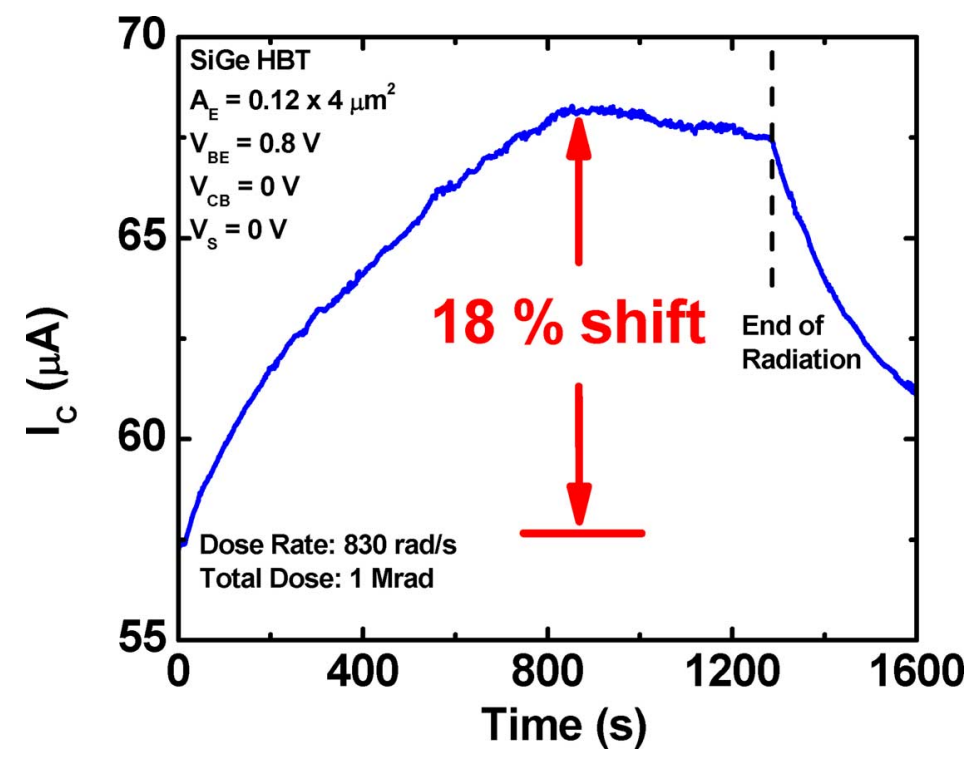

Fig. 4. Collector and base currents of a SiGe HBT under irradiation of 1 Mrad. Radiation stopped at 1,275 s, but biasing continued until a total of $1,600 \mathrm{~s}$ was reached.

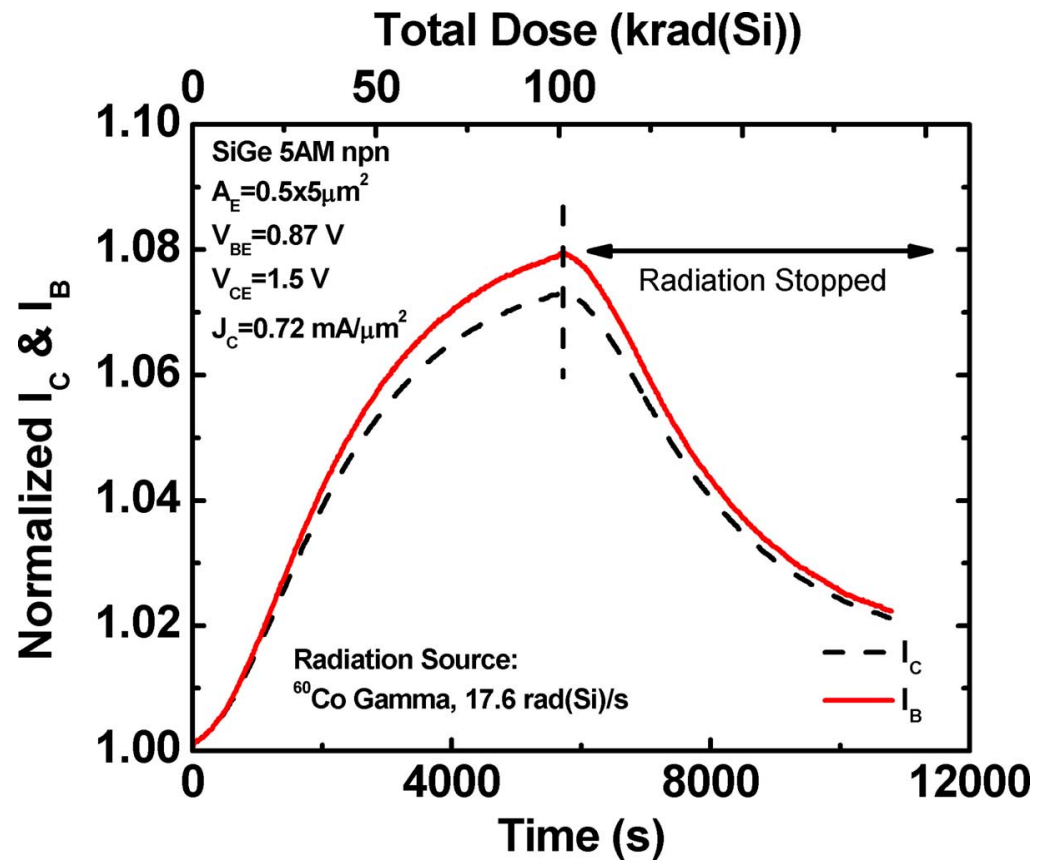

Fig. 5. Normalized collector and base current during gamma TID testing. The device was forward-biased, and the dose rate was set to be $17.6 \mathrm{rad}(\mathrm{Si}) / \mathrm{s}$. After 100 krad TID was reached in approximately $6000 \mathrm{~s}$, the device was continued to be biased for another $5000 \mathrm{~s}$.

(BGR) were also examined: they were fabricated in IBM SiGe 5AM technology. The $63.3 \mathrm{MeV}$ proton irradiation was performed at Crocker Nuclear Laboratory at the University of California at Davis, which achieves $15 \%$ uniformity over a $2.0 \mathrm{~cm}$ radius (the dosimetry system is accurate to about 10\%) [13]. Gamma TID irradiation was performed using a ${ }^{60} \mathrm{Co}$ source, and the dosimetry was measured using chamber probes.

\section{EXPERIMENTAL RESULTS: SiGE HBTS}

For all radiation experiments discussed in this paper, the $\mathrm{SiGe}$ HBTs were biased in forward-active mode, as they would be used in a circuit. The $I_{C}$ and $I_{B}$ before, during, and after irradiation were recorded. Shown on Fig. 2 is an $8 \mathrm{WL}$ device during proton irradiation, at dose rate of $830 \mathrm{rad}(\mathrm{Si}) / \mathrm{s}$. Instead of measuring the Gummel characteristics, $I_{B}$ and $I_{C}$ are sampled at real-time at a fixed $V_{B E}$ and $V_{C B}$. While they are constant before irradiation, the currents suddenly increase sub-linearly during irradiation, and decay logarithmically afterwards. The current gain of the device is plotted in Fig. 3, and clearly it remains constant throughout the experiment. Fig. 4 shows another SiGe HBT irradiated up to a total dose of $1 \mathrm{Mrad}(\mathrm{Si})$. The $I_{C}$ increases up to a certain level, and then begins to recover, even during irradiation. The recovery process accelerates once 


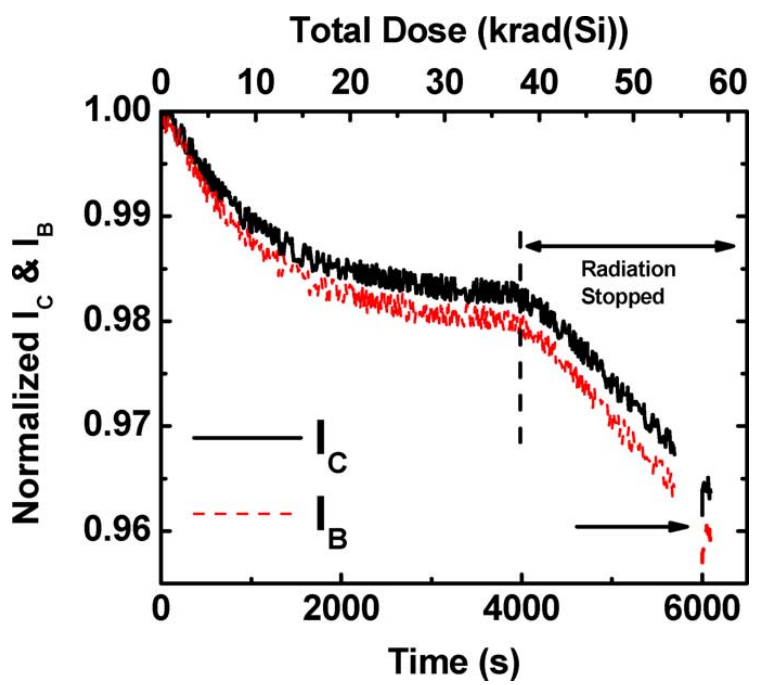

Fig. 6. Normalized collector and base currents during gamma TID testing. The device was forward-biased with dose rate $9.5 \mathrm{rad}(\mathrm{Si}) / \mathrm{s}$.

irradiation was halted. A maximum of $18 \%$ excess $I_{C}$ was observed, which is clearly significant. For comparison, SiGe HBTs were also tested using traditional protocols, in which devices were taken out of the radiation chamber after exposure, and then re-measured. Post-irradiation collector current data in this case showed no observable change compared to pre-irradiation data, indicating that traditional test protocols and our present test protocol give different results.

Since one dose rate is inadequate in determining radiation hardness, and $830 \mathrm{rad} / \mathrm{s}$ is higher than what is suggested in MIL-STD-883G Test Method 1019.7[1], ${ }^{60}$ Co gamma irradiation was used for lower dose rate experiments. The devices used in the gamma irradiation experiments were 5AM SiGe HBTs, and the dose rate varied from $17 \mathrm{rad}(\mathrm{Si}) / \mathrm{s}$ to $0.23 \mathrm{rad}(\mathrm{Si}) / \mathrm{s}$. Shown in Fig. 5 are the normalized $I_{C}$ and $I_{B}$ during gamma irradiation, at dose rate of $16.6 \mathrm{rad}(\mathrm{Si}) / \mathrm{s}$. Similar to the proton results, the currents increase sub-linearly during irradiation to about $8 \%$, and then recovered afterwards. Compared with the $830 \mathrm{rad}(\mathrm{Si}) / \mathrm{s}$ proton experiment, the $16.6 \mathrm{rad}(\mathrm{Si}) / \mathrm{s}$ gamma $I_{B}$ and $I_{C}$ changes much more gradually.

Going to even lower dose rates reveals quite different results. Shown in Fig. 6 are normalized $I_{B}$ and $I_{C}$ at a dose rate of $9.5 \mathrm{rad}(\mathrm{Si}) / \mathrm{s}$. The currents unexpectedly decreased during irradiation, and even continued to drop afterwards the exposure was stopped. The degradation rate during the annealing period is actually higher than during irradiation. Previous gamma irradiation studies have also reported shifts in $I_{C}$ [14], which was attributed to ambient temperature change. In order to ensure that shifts in current are not due to any temperature fluctuation (perhaps during irradiation), these devices were monitored continuously for half an hour following irradiation. To further confirm the repeatability of the degradation, the measurement was stopped for approximately 5 minutes and then continued. As can be seen in Fig. $6, I_{C}$ and $I_{B}$ shifts still exist, continuing from where they would be had the measurement not been stopped.

Fig. 7 shows the dynamics of $I_{C}$ across a wide range of dose rates. At dose rates above $11.8 \mathrm{rad}(\mathrm{Si}) / \mathrm{s}, I_{C}$ shows a sub-

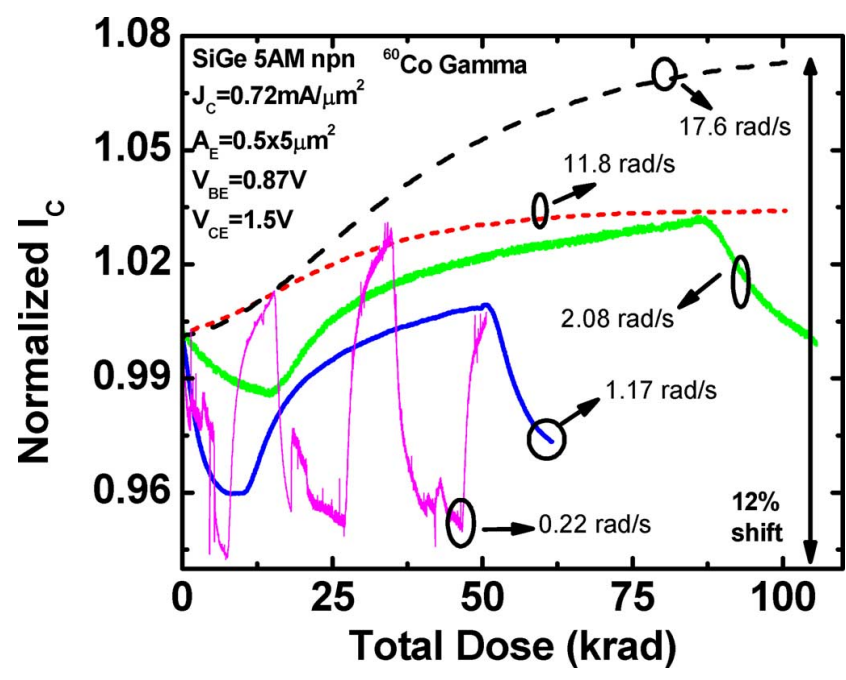

Fig. 7. Normalized collector current during gamma TID testing. Each device was forward-biased at the same $V_{B E}$ and $V_{C E}$, and the dose rates were 17.6, $11.8,2.08,1.17$, and $0.22 \mathrm{rad}(\mathrm{Si}) / \mathrm{s}$.

linear increase as a function of total dose; however, below 2.08 $\operatorname{rad}(\mathrm{Si}) / \mathrm{s}$ the currents can be seen to fluctuate, and the frequency of the fluctuation is higher for lower dose rates. As can be seen in Fig. 7, a maximum of $12 \%$ shift in $I_{C}$ has been observed in gamma irradiation testing, which is clearly a significant change.

In all of the experiments, pre- and post-irradiation Gummel characteristics were measured. During most of the experiments, the devices continued to be biased for a long period after the radiation exposure ceased, in order to monitor the annealing behavior, and as can be seen in Figs. 4 and 5, most of the $I_{C}$ and $I_{B}$ shift would have been recovered by then. For these samples, the pre- and post-irradiation Gummel curves nearly overlap, with a maximum of $1 \%$ shift in the currents. For a few low-dose rate experiments, the post-rad Gummel curves were measured immediately $(<10 \mathrm{~s})$ after irradiation. An example is shown in Fig. 8, where the device was exposed to $1.17 \mathrm{rad}(\mathrm{Si}) / \mathrm{s}$ gamma irradiation. Indeed, the pre- and post-irradiation Gummel curves differ by $12 \%$ across $V_{B E}$, and the current shifts have nearly the same $1 k T$ slope as the original $I_{B}$ and $I_{C}$.

\section{Circuit Hardness Assurance}

If the operating point ( $\mathrm{V}$ and I bias) of a transistor shifts during radiation exposure, circuit performance can be potentially compromised. To observe the potential circuit impact of the observed changes in bias currents $\left(I_{\text {bias }}\right), \mathrm{X}$-band (8-12 $\mathrm{GHz}$ ) phase shifters from a radar chip set were irradiated. The $I_{\text {bias }}$ of a SiGe HBT phase shifter is shown to increase by about $1 \mathrm{~mA}$ during exposure (Fig. 9). The SiGe phase shifter is biased using a set of current mirrors, with external reference current $\left(I_{e x t}\right)$ supplied through the Agilent 4155 . The voltage at this terminal, $V_{e x t}$, plotted also in Fig. 9, is essentially the $V_{B E}$ of the mirroring SiGe HBT. The decrease of $V_{\text {ext }}$ shows the same pattern as the excess $I_{C}$ described above, with the result being that less $V_{B E}$ is required to supply the same current. For the CMOS $\mathrm{X}$-band phase shifters, $I_{\text {bias }}$ increased from less than $0.3 \mu \mathrm{A}$ 


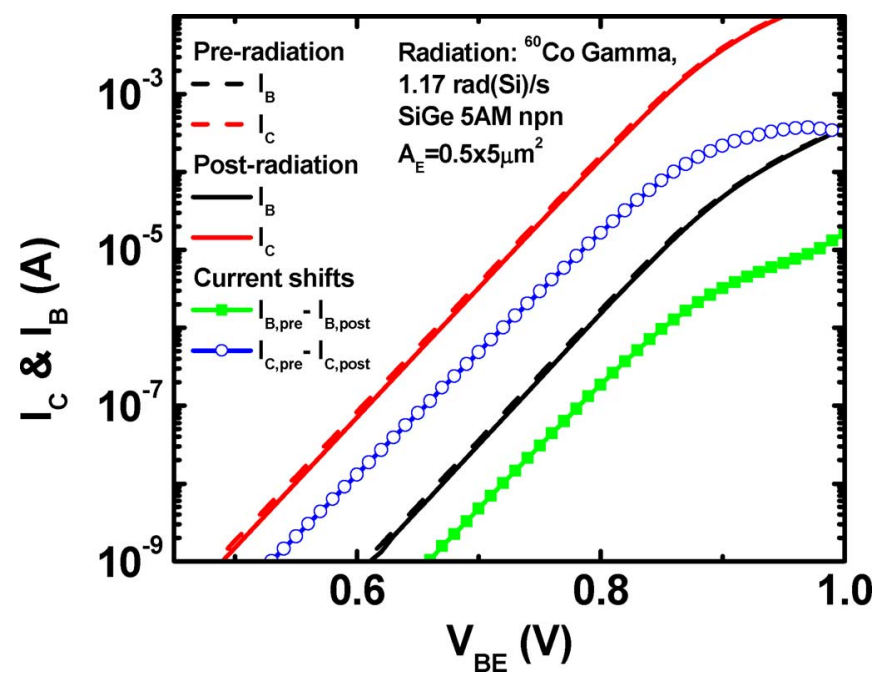

Fig. 8. Pre- and post-radiation Gummel characteristics. The post-irradiation measurements were done almost immediately after irradiation. Also shown are the $I_{B}$ and $I_{C}$ current shifts.
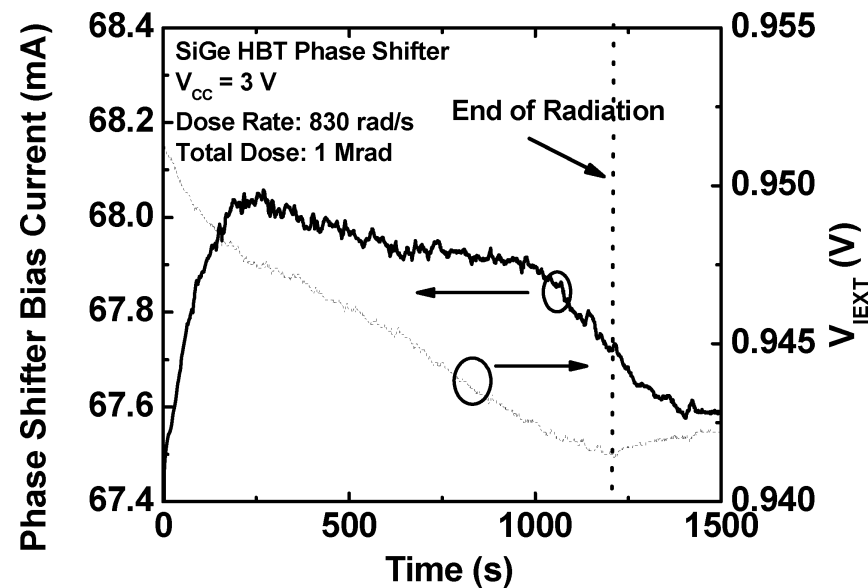

Fig. 9. Bias current shift of an X-band SiGe HBT phase shifter during proton irradiation. The circuit was biased using current mirrors with $\mathrm{I}_{\mathrm{EXT}}=-1.85 \mathrm{~mA}$. V $\mathrm{V}_{\mathrm{IEXT}}$ (also plotted) is the voltage at this node.

to about $3 \mu \mathrm{A}$ during irradiation (Fig. 10). This is a large percentage change; however, in terms of actual leakage magnitude, it is only $2.7 \mu \mathrm{A}$.

Precision voltage references are critical components for virtually all analog/RF circuitry because they set the biasing conditions for many circuits in integrated electronic systems. SiGe bandgap references (BGRs), such as the ones used in a newly-developed data acquisition system for NASA lunar missions [15], were used for this experiment. The operating current and output voltage $\left(V_{\text {out }}\right)$ of these SiGe BGRs were monitored during irradiation. Irradiation was halted for a period of approximately 150 seconds before resuming again. The percentage change of $I_{\text {bias }}$ is plotted in Fig. 11 and only a 3\% change in $I_{\text {bias }}$ was observed, with $V_{\text {out }}$ altered by about $3 \mathrm{mV}$, not insignificant for some applications (e.g., data converters). Fig. 11 shows that the BGR is still functioning correctly.

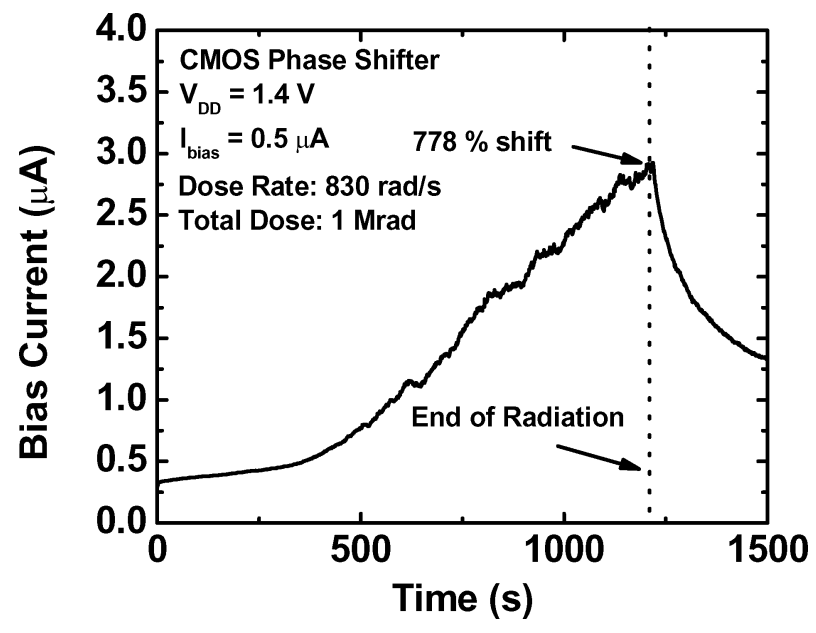

Fig. 10. Bias current shift of X-band CMOS phase shifter during proton irradiation.

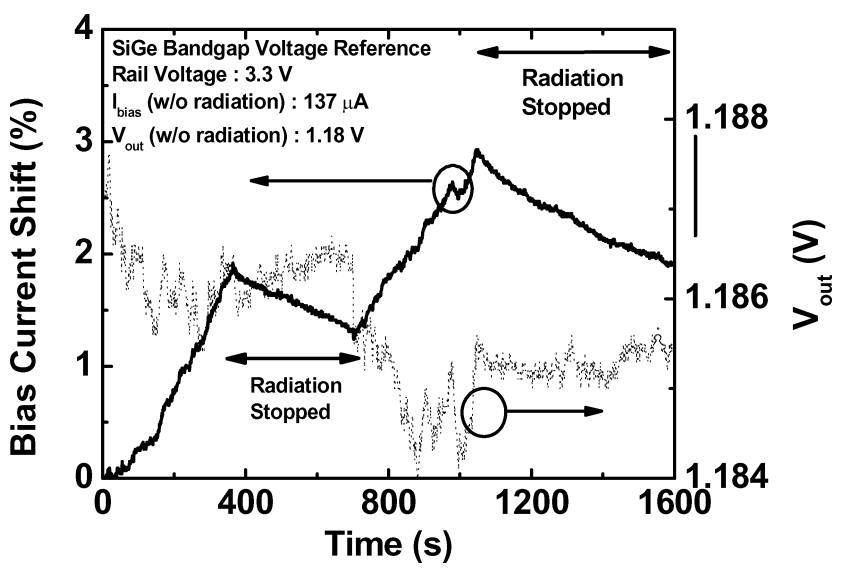

Fig. 11. Bias current shift in a SiGe voltage reference during proton irradiation. The circuit was irradiated to $500 \mathrm{krad}(\mathrm{Si})$. Also plotted is the output voltage shift during irradiation.

\section{DISCUSSION}

Based on these TID test results, several issues can be identified. First, we focus on the damage mechanisms behind the observed collector current changes of these SiGe HBTs, and then we evaluate the hardness assurance of SiGe BiCMOS processes from our results. Finally, we offer improved test protocol recommendations. Base current degradation due to traps has been studied extensively [3]-[5] and will not be discussed here. Instead, collector current degradation will be the focus here.

\section{A. Damage Mechanisms}

$I_{C}$ shifts due to irradiation have been observed from the very first experimental studies of first generation SiGe HBTs. In [7] SiGe HBTs were gamma-irradiated up to $6 \mathrm{Mrad}$, and the collector current was observed to increase first and then decrease after $1 \mathrm{Mrad}$. It was observed that the devices showed approximately a $20 \%$ change in $I_{C}$ (the devices were irradiated at dose rate of $48 \mathrm{rad}(\mathrm{Si}) / \mathrm{s})$. In [16], $I_{C}$ was observed to fluctuate ( $\sim 30 \%$ ) with neutron irradiation, and seemed to be decreasing with increasing dose. 


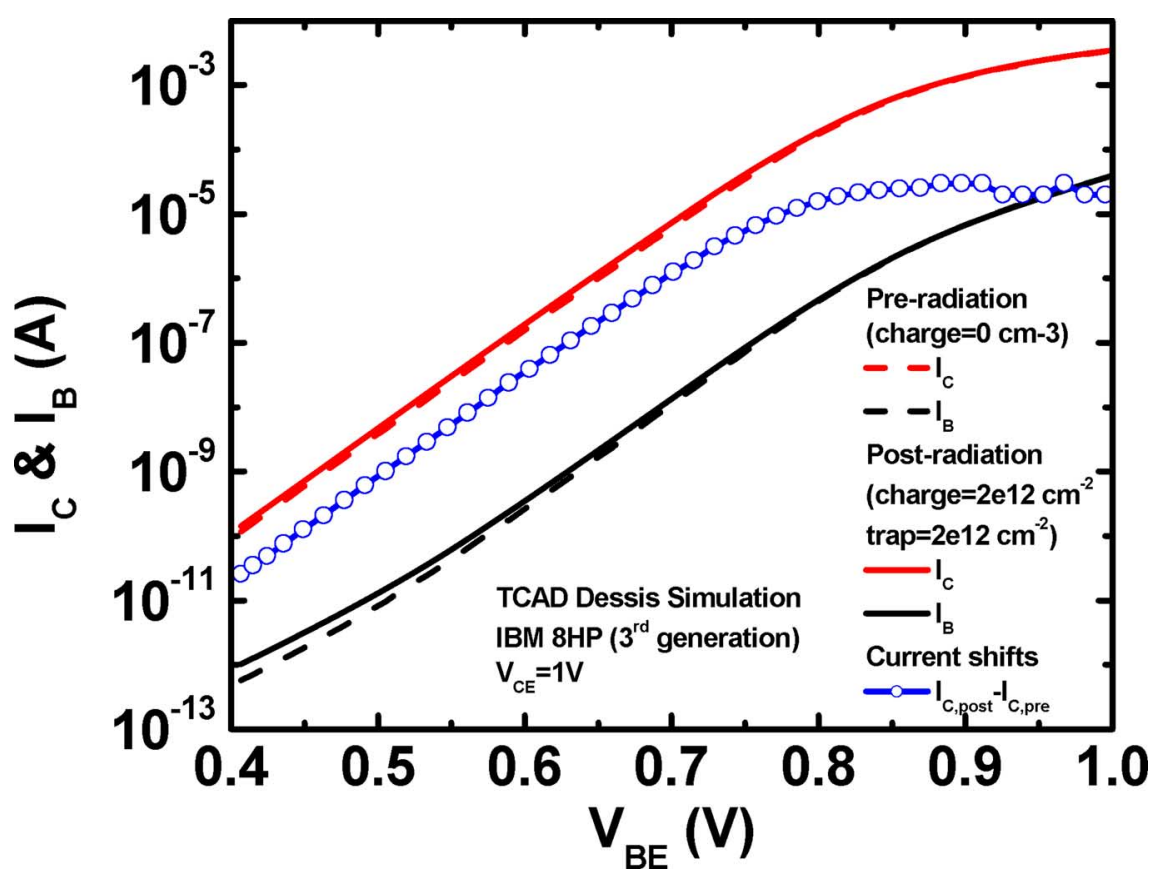

Fig. 12. TCAD Dessis Simulation of $I_{C}$ and $I_{B}$ shift. The traps and charges at the interface increase the base and collector leakage currents. The excess $I_{C}$ is also shown.

A general framework for understanding the physical origin of the excess $I_{C}$ can be taken from [17], which notes that radiation-induced excess $I_{C}$ can be caused by an inversion layer generated under the EB spacer during irradiation. This inversion layer increases the effective emitter area, thus increasing the overall $I_{C}$. This is in agreement with results shown in Figs. 4 and 5. Measurements from [17] showed excess $I_{C}$ in the range of $15 \%$, which is close to our data.

However, one distinct difference between our measurements and the previous finding is that both collector and base current are shifting, thus maintaining a nearly constant current gain. Simulations [17], [18] have confirmed that the inversion layer alone could not affect the base current, since the base current predominately depends on the emitter Gummel number. TCAD simulations have been performed to determine if charges and/or traps are located in the polysilicon/silicon, silicon/silicon-dioxide, and silicon/silicon-germanium interfaces. Perhaps a possible explanation would be that during irradiation both interface charge and trap density increases; the former creates an inversion layer to increase $I_{C}$, whereas the latter increases the SRH recombination current in $I_{B}$. For $I_{B}$ to increase as a $1 k T$ slope (as seen in Fig. 8), the traps cannot be midgap traps but instead should be shallow traps near the conduction band. TCAD simulations using an 8HP calibrated model demonstrates that this can cause both $I_{C}$ and $I_{B}$ to shift in the same direction (see Fig. 12). In our simulations the interface charge and trap density was set to be $2 \times 10^{12} \mathrm{~cm}^{-2}$, with the trap energy located $0.1 \mathrm{eV}$ below the conduction band.

It was reported in [19] that a $1 k T$ slope $I_{B}$ leakage can be created due to midgap traps, provided there is enough charge at the interface; however this would also generate a large $2 k T I_{B}$ leakage, which is not the case in our measurements.
Another major difference between our measurement results and previous studies is the significant annealing observed in $I_{C}$. From a wide range of dose rates used in this study, we find that the annealing rate is observed to change with dose rate by nearly 2 orders of magnitude; the higher the dose rate, the faster the annealing after irradiation.

The fluctuation of $I_{C}$ seen at low dose rates and the annealing characteristics (Figs. 2 and 7) are very characteristic of results shown in [20], [21]. In [21], for irradiated MOSFETs, the gate interface traps were observed to increase when positively biased and decrease once negatively biased. This bias-dependence of the traps was determined to be due to protons inside the oxide. When positively biased, the protons move towards the interface, and thus the trap generation process dominates. The sub-linear behavior of trap formation was most likely due to the diffusion process of hydrogen molecules. Once the bias becomes negative, protons are moved away from the interface, causing the trap annealing process to dominate.

However, as opposed to results in [21], where the gate oxide was biased, the EB spacer inside SiGe HBTs is inherently unbiased. For protons to move towards and away from the interface, the electric field inside the spacers must also change. To the authors' knowledge, no such explanation exists in the literature.

\section{B. Hardness Assurance of SiGe BiCMOS Processes}

Even though the damage mechanism in the present investigation is not yet well-understood, it can be seen from test results that $I_{C}$ generally shifts around $10 \%$ to $12 \%$, with a maximum shift (up to a TID of $1 \mathrm{Mad}(\mathrm{Si})$ ) of $18 \%$. In the context of RF circuit design, where current densities are carefully fixed, this excess $I_{C}$ could only negatively affect circuit performance. If devices are biased at high $J_{C}$ to achieve the highest possible 
gain, the excess $I_{C}$ could cause premature base pushout and impedance mismatch, which will degrade RF performance.

In the context of analog circuit design, in situations where a given circuit specification is very demanding, excess $I_{C}$ could pose a serious problem. As can be seen in the BGRs presented here, bias currents increased by no more than $3 \%$, but changes in the output voltage can in certain circumstances be of serious concern. We suspect that the importance of our observations will depend on the specific application (hence circuit) in question, and for that reason alone should be carefully assessed.

\section{Hardness Assurance Testing Recommendations}

In previous studies, post-irradiation characterization of devices was performed by removing the packages from the radiation beam, taking the DUTs to a separate, radiation-free environment, and then re-measuring them. This may require a period of several minutes (or longer) in order to wait for the background radiation level in the chamber to become safe enough to enter [8]. For high dose rates, this waiting period can be significant, since most of the excess $I_{C}$ would have already recovered. For low dose rates, the recovery rate is quite slow, and therefore the waiting period may not cause significant changes. However, since the excess $I_{C}$ is fairly small (in the range of a few percent) it seems that the waiting period should be as short as possible, regardless of dose rate. Considering the small variation of this damage and the recommendation of Section 3.10 of MIL-STD-883G Test Method 1019.7, we believe that monitoring the bias currents continuously during irradiation is the best way to fully characterize the extent of the radiation damage in SiGe HBTs. It seems that revised TID hardness assurance protocols may be needed to meet the challenge of capturing damage characteristics for highly scaled, modern transistors.

\section{SUMMARY}

We have investigated the applicability of current TID test protocols in the context of advanced transistor technologies such as SiGe HBTs. Annealing effects were observed immediately after irradiation, leading to a discrepancy between the actual damage measured real-time and that measured after a time delay. Thus, for hardness assurance we recommend monitoring the devices continuously before, during, and after irradiation. For SiGe HBTs, an unexpected excess collector current is observed during total dose irradiation, which was shown to have a different response to dose rate. We attribute this phenomenon to radiation-induced charge, which creates an inversion layer and increase the collector current, and interface traps, which increase the base recombination current. Our TCAD simulations are able to qualitatively match the measurement data. The impact of our observations is very likely circuit specific and application dependent and thus warrants careful assessment.

\section{ACKNOWLEDGMENT}

The authors are grateful to K. LaBel, L. Cohn, T. Ward, and S. Brown for their contributions.

\section{REFERENCES}

[1] MIL-STD 883, Test Method 1019, [Online]. Available: http:// www.dscc.dla.mil/Programs/MilSpec/listDocs.asp?BasicDoc=MILSTD-883

[2] T. R. Oldham, F. B. McLean, H. E. Boesch Jr., and J. M. McGarrity, "An overview of radiation-induced interface traps in MOS structures," Semiconductor Sci. Technol., vol. 4, no. 12, pp. 986-999, 1989.

[3] A. Sutton, B. M. Haugerud, A. P. G. Prakash, B. Jun, J. D. Cressler, C. J. Marshall, P. W. Marshall, R. Ladbury, F. Guarin, and A. J. Joseph, "A comparison of gamma and proton radiation effects in $200 \mathrm{GHz} \mathrm{SiGe}$ HBTs," IEEE Trans. Nucl. Sci., vol. 52, no. 6, pp. 2358-2365, 2005.

[4] M. Bellini, B. Jun, A. K. Sutton, A. C. Appaswamy, P. Cheng, J. D. Cressler, P. W. Marshall, R. D. Schrimpf, D. M. Fleetwood, B. El-Kareh, S. Balster, P. Steinmann, and H. Yasuda, "The effects of proton and X-ray irradiation on the DC and AC performance of complementary (npn+pnp) SiGe HBTs on thick-film SOI," IEEE Trans. Nucl. Sci., vol. 54, pp. 2245-2250, 2007.

[5] R. N. Nowlin, D. M. Fleetwood, R. D. Schrimpf, R. L. Pease, and W. E. Combs, "Hardness-assurance and testing issues for bipolar/BiCMOS devices," IEEE Trans. Nucl. Sci., vol. 40, pp. 1686-1693, 1993.

[6] A. Wei, S. L. Kosier, R. D. Schrimpf, W. E. Combs, and M. DeLaus, "Excess collector current due to an oxide-trapped-charge-induced emitter in irradiated NPN BJT's," IEEE Trans. Nucl. Sci., vol. 42, pp. 923-027, 1995.

[7] J. A. Babcock, J. D. Cressler, L. S. Vempati, S. D. Clark, R. C. Jaegar, and D. L. Harame, "Ionizing radiation tolerance of high-performance SiGe HBT's grown by UHV/CVD," IEEE Trans. Nucl. Sci., vol. 42, pp. 1558-1566, 1995.

[8] J. A. Babcock, J. D. Cressler, L. S. Vempati, S. D. Clark, R. C. Jaegar, and D. L. Harame, "Ionizing radiation tolerance and low-frequency noise degradation in UHV/CVD SiGe HBTs," IEEE Electron Device Lett., vol. 16, pp. 351-353, 1995.

[9] D. L. Hansen, S. Pong, R. Rosenthal, and J. Gorelick, "Total ionizing dose testing of SiGe 7HP discrete heterojunction bipolar transistors for ELDRS effects," in Proc. IEEE Radiation Effects Data Workshop, 2007, pp. 215-220.

[10] L. Lanzerotti, N. Feilchenfeld, D. Coolbaugh, J. Slinkman, P. Gray, D. Sheridan, J. Higgins, W. Hodge, M. Gordon, T. Larsen, M. Gautsch, P. Lindgren, R. Murty, J. Rascoe, K. Watson, T. Stamper, E. Eshun, J. He, K. Downes, R. Rassel, J. Greco, B. Labelle, S. Sweeney, K. Stein, R. Bolam, K. Vaed, B. Omer, A. Joseph, S. St Onge, and J. Dunn, "A low complexity $0.13 \mu \mathrm{SiGe}$ BiCMOS technology for wireless and mixedsignal applications," in Proc. IEEE Bipolar/BiCMOS Circuits Technol. Conf., 2004, pp. 237-240.

[11] D. L. Harame, D. C. Ahlgren, D. D. Coolbaugh, J. S. Dunn, G. G. Freeman, J. D. Gillis, R. A. Groves, G. N. Hendersen, R. A. Johnson, A. J. Joseph, S. Subbanna, A. M. Victor, K. M. Watson, C. S. Webster, and P. J. Zampardi, "Current status and future trends of SiGe BiCMOS technology," IEEE Trans. Electron Devices, vol. 48, pp. 2575-2594, 2001.

[12] B. Jagannathan, M. Khater, F. Pagette, J. S. Rieh, D. Angell, H. Chen, J. Florkey, F. Golan, D. R. Greenberg, R. Groves, S. J. Jeng, J. Johnson, E. Mengistu, K. T. Schonenberg, C. M. Schnabel, P. Smith, A. Stricker, D. Ahlgren, G. Freeman, K. Stein, and S. Subbanna, "Self-aligned SiGe NPN transistors with $285 \mathrm{GHz}$ f/sub MAX/ and $207 \mathrm{GHz}$ / sub T/ in a manufacturable technology," IEEE Electron Device Lett., vol. 23, pp. 258-260, 2002.

[13] K. M. Murray, W. J. Stapor, and C. Casteneda, "Calibrated charged particle radiation system with precision dosimetric measurements and control," Nuclear Instrum. Methods Phys. Res., vol. 281, no. 3, pp. 616-621, 1989.

[14] J. D. Cressler, R. Krithivasan, A. K. Sutton, J. E. Seiler, J. F. Krieg, S. D. Clark, and A. J. Joseph, "The impact of gamma irradiation on SiGe HBTs operating at cryogenic temperatures," IEEE Trans. Nucl. Sci., vol. 50, pp. 1805-1810, 2003.

[15] R. Berger, R. Garbos, J. D. Cressler, M. Mojarradi, L. Peltz, B. Blalock, W. Johnson, G. Niu, F. Dai, A. Mantooth, J. Holmes, M. Alles, and P. McClusky, in Proc. IEEE Aerospace Conf., 2008, pp. 1-12.

[16] J. M. Roldan, W. E. Ansley, J. D. Cressler, S. D. Clark, and D. Ngugen-Ngoc, "Neutron radiation tolerance of advanced UHV/CVD SiGe HBT BiCMOS technology," IEEE Trans. Nucl. Sci., vol. 44, no. 6, pp. 1965-1973, 1997.

[17] A. Wei, S. L. Kosier, R. D. Schrimpf, W. E. Combs, and M. DeLaus, "Excess collector current due to an oxide-trapped-charge-induced emitter in irradiated NPN BJT's," IEEE Trans. Nucl. Sci., vol. 42, pp. 923-927, 1995. 
[18] P. S. Charkraborty, A. Appaswamy, P. K. Saha, N. K. Jha, J. D. Cressler, and H. Yasuda, "Mixed-mode stress degradation mechanisms of pnp SiGe HBTs," in Pro. Int. Res. Phys. Symp., 2009, pp. 83-88.

[19] S. L. Kosier, R. D. Schrimpf, R. N. Nowlin, D. M. Fleetwood, M. DeLaus, R. L. Pease, W. E. Combs, A. Wei, and F. Chai, "Charge separation for bipolar transistors," $v$, vol. 40, pp. 1276-1285, 1993.
[20] S. N. Rashkeev, D. M. Fleetwood, R. D. Schrimpf, and S. T. Pantelides, "Effects of hydrogen motion on interface trap formation and annealing," IEEE Trans. Nucl. Sci., vol. 51, pp. 3158-3165, 2004.

[21] D. M. Fleetwood, W. L. Warren, P. S. Schwank, M. R. Shanefelt, and L. C. Riewe, "Effects of interface traps and border traps on MOS postirradiation annealing response," IEEE Trans. Nucl. Sci., vol. 42, pp. 1698-1706, 1995. 